\title{
Timely and Efficient Chewing and Mashing
}

National Cancer Institute

\section{Source}

National Cancer Institute. Timely and Efficient Chewing and Mashing. NCI Thesaurus.

Code $C 127198$

A finding of timely and efficient chewing and mashing prior to swallowing. 